\title{
ARTICLE
}

\section{Parity of esteem: addressing the inequalities between mental and physical healthcare}

\author{
Alex J. Mitchell, Sheila Hardy \& David Shiers
}

\begin{abstract}
Alex J. Mitchell is Professor of Psycho-oncology and Liaison Psychiatry at the University of Leicester and a consultant psychiatrist with Leicester Partnership NHS Trust. Sheila Hardy is a Senior Research Fellow with Northamptonshire Healthcare NHS Foundation Trust, an honorary senior lecturer at University College London, a post-graduate nurse educator with the Charlie Waller Memorial Trust and a Visiting Fellow at the University of Northampton. David Shiers is a former general practitioner in North Staffordshire. He is now an honorary reader in the Division of Psychology and Mental Health at the University of Manchester and an honorary research consultant to the Psychosis Research Unit at Greater Manchester West Mental Health Foundation Trust. He is a topic expert for the National Institute for Health and Care Excellence (NICE) guidance on psychosis and schizophrenia in children and young people, and a board member of National Collaborating Centre for Mental Health (NCCMH). The views expressed in this article are his personal views and not necessarily those of NICE or NCCMH.

Correspondence Professor

SUMMARY

Parity of esteem means valuing mental health as much as physical health in order to close inequalities in mortality, morbidity or delivery of care. There is clear evidence that patients with mental illness receive inferior medical, surgical and preventive care. This further exacerbated by low help-seeking, high stigma, medication sideeffects and relatively low resources in mental healthcare. As a result, patients with severe mental illness die 10-20 years prematurely and have a high rate of cardiometabolic complications and other physical illnesses. Many physical healthcare guidelines and policy recommendations address parity of esteem, but their implementation to date has been poor. All clinicians should be aware that inequalities in care are adversely influencing mental health outcomes, and managers, healthcare organisations and politicians should provide resources and education to address this gap.

\section{LEARNING OBJECTIVES}

- Understand the concept of parity of esteem

- Be aware of the current inequalities in mental healthcare

- Appreciate how parity of esteem may be improved

\section{DECLARATION OF INTEREST}

None
\end{abstract}

Alex J. Mitchell, Department of

Psycho-Oncology, Leicester General Hospital, Gwendolen Road, Leicester LE5 4PW, UK. Email: ajm80@le.ac.uk

Copyright and usage

(C) The Royal College of Psychiatrists 2017.
Findings from the Global Burden of Disease Study 2010, a comprehensive analysis of burden for 291 diseases and injuries and 67 risk factors, showed that mental and substance use disorders were responsible for $7.4 \%$ of global disability-adjusted life-years (DALYs) and $22.9 \%$ of global years lived with disability (YLDs), ranking 5th and 1st respectively (Whiteford 2013, 2015). Some argue that these are underestimates and figures should be $13.0 \%$ of DALYs and $32.4 \%$ of years lived with disability (Vigo 2016). Furthermore, there are important regional variations. Mental disorder DALYs are higher in Europe and highest in North Africa and the Middle East; substance use disorder DALYs are highest in Eastern Europe and Central Asia (Whiteford 2015). Yet despite this burden, only a minority of people with mental disorders in high-income counties receive adequate treatment (Kessler 2001). Treatment in low- and middleincome countries is much lower still (World Health Organization 2013). This treatment gap exists because of high stigma, low help-seeking, low recognition, low resources and lack of acceptable treatments offered promptly (Clement 2015). Many sufferers do not recognise their own condition as warranting attention, do not think that treatment will be effective and wait 8 years or more to ask for help (Kessler 2001).

Equally alarming is that patients with current or past mental disorders have accelerated mortality. One meta-analysis showed that the relative risk of mortality in those with mental disorders is 2.22 (95\% CI 2.12-2.33), accounting for a median 10 years of life lost and $14.3 \%$ of deaths worldwide (8 million deaths each year) (Walker 2015). At least two-thirds of deaths among people with mental disorders are due to natural causes and about one in five to unnatural ones. Although progress has been made in reducing unnatural deaths (violent deaths and suicide) in this group, between 1982 and 2006 the risk of premature death due to cardiovascular disease more than doubled in comparison with that of the general population (Brown 2010). The mental health mortality gap between observed and expected deaths has not improved despite increased recognition of the importance of physical comorbidity. Many factors, including background, lifestyle, economic disadvantage, unhelpful health behaviours (especially smoking), and difficulties accessing and adhering to medical treatments, are thought to contribute to premature mortality in severe mental illness (SMI) such as schizophrenia (Olfson 2015). Mental health professionals may have unwittingly increased the risk of medical conditions, and possibly mortality, by recommending a variety of psychotropic drugs that contribute to cardiovascular disease, diabetes and obesity (Mitchell 2013a). However, the link between antipsychotics and premature mortality is complex (Torniainen 2015). Adults with mental illness are also less likely than age-matched peers to receive 
adequate treatment for major medical conditions, which may compound risk of premature mortality (Mitchell 2012a; Woodhead 2016). In some cases, unequal quality of healthcare provision may explain much of the subsequent excess mortality (Mitchell 2010; Druss 2011). Many people with physical health problems also have mental health problems and vice versa, yet medical science and training often fail to integrate these and teach the importance of comorbidity. Indeed, in all settings, but perhaps particularly in primary care, it is multiple morbidity that remains an unresolved challenge and a key barrier to achieving parity (Mujica-Mota 2015; Paddison 2015). The aim of this article is to review the concept of parity of esteem as applied to mental-physical comorbidity.

\section{The concept of parity of esteem}

Given the headlines that mental disorders feature greater illness burden, greater mortality but lower treatment uptake than physical disorders, many have called for at least comparable attention to that given to major medical disorders. This is called parity of esteem: an initiative that recognises and aims to address inequalities between physical and mental healthcare, including preventable premature deaths (the mortality gap), lower-quality medical care (the treatment gap) and inferior resources for mental healthcare relative to the impact of mental health problems (the funding gap). In simple terms, parity of esteem refers to the concept that society should value mental health to the same degree that it values physical health.

In the USA, parity became law in 1996 under the Mental Health Parity Act (MHPA) and later under the Mental Health Parity and Addiction Equity Act (MHPAEA) of 2008. The MHPAEA requires that limits on mental health insurance benefits be no lower than any such limits on medical and surgical benefits. Fleischhacker et al (2008) suggested that parity in healthcare for people with mental illness should be regarded as a basic human right.

In the UK, the Equality Act 2010 placed a legal responsibility on health services to make reasonable adjustments to ensure that people with SMI are not disadvantaged compared with the general population in accessing healthcare. Parity was formally proposed in the 2011 governmental mental health strategy for England 'No Health without Mental Health'. However, the concept was not well understood and implementation was slow. Consequently, the Department of Health asked the Royal College of Psychiatrists to develop a vision for 'parity of esteem' (Box 1), which was disseminated in 2013 (Royal College of Psychiatrists 2013). In February 2016, NHS England's Mental Health Taskforce produced a
BOX 1 Royal College of Psychiatrists' definition of parity of esteem

Compared with physical healthcare, mental healthcare should be characterised by:

- equal access to effective, safe care

- equal efforts to improve the quality of care

- the allocation of resources on a basis commensurate with need

- equal status within healthcare education and practice

- equally high aspirations for patients

- equal status in the measurement of health outcomes

- holistic, integrated care with mental health considered alongside physical health

(Royal College of Psychiatrists 2013)

report and recommendations on mental healthcare (Mental Health Taskforce 2016).

Parity of esteem can be viewed from the perspective of patients (the right to equal standards of quality of care in both mental and physical health), from the perspective of clinicians (equal focus on both mental and physical health) and from the perspective of commissioners (providers should devote the same time and resources to improving mental health as they do to physical health). In 2014, NHS England established a Parity of Esteem Programme. Mainly aimed at commissioners, its purpose is to focus effort and resources on improving clinical services and health outcomes (NHS England 2014). This has led to a call to action and payment incentives through the Commissioning for Quality and Innovation (CQUIN) framework to improve physical health in severe mental illness. In 2014, the British Medial Association (BMA) published a report on parity of outcomes that sets out steps that need to be taken in the following key areas (BMA Science and Education Department 2014):

- promoting prevention and early intervention strategies

- delivering ‘joined-up' care

- enhancing training and workforce planning

- encouraging a greater research focus on mentalphysical multimorbidity.

\section{Examples of gaps in parity of esteem (inequalities in care)}

\section{Inequalities in mass screening}

Inequalities in medical care of people with mental illness have been documented in preventive mass screening procedures. Lord et al (2010) reviewed 61 comparisons of preventive care, from 26 studies across Europe and North America, in individuals 
with and without psychiatric illness. Twentyseven comparisons revealed inferior preventive healthcare in those with mental illness; conversely, 10 suggested superior preventive healthcare, and 24 reached inconclusive findings. However, in a more refined analysis focusing on mammography, Mitchell and colleagues found significantly reduced rates of mammography screening in women with mental illness $(\mathrm{OR}=0.71,95 \%$ CI $0.66-0.77)$, particularly SMI (OR $=0.54,95 \%$ CI $0.45-0.65)$, not due to distress alone, but rather related to the diagnosis of mental illness itself (Mitchell 2014).

\section{Inequalities in dental care}

People with SMI have higher rates of smoking and higher sugar intake (e.g. carbonated drinks). Patients brush their teeth infrequently and attend the dentist less often than the general population (McCreadie 2004). A review of 57 studies reported a $61 \%$ lifetime prevalence of poor oral health and twice the rate of decayed, missing or filled teeth (Wey 2016). People with SMI have three times the odds of having lost all their teeth than the general community (Kisley 2011).

\section{Inequalities in medical care}

Several valuable reviews have highlighted the extent of inequalities in medical care (Mitchell 2009; Lawrence 2010; Moore 2015). Inequalities are most notable in cardiovascular and diabetes care: for example, in primary care those with schizophrenia are less likely to have a record of cardiovascular disease (suggesting under-recognition) (Smith 2013). One explanation is that these inequalities result from low attendance or adherence. Yet many people with mental ill health, in particular those with depressive disorders, seek help more often and use more general healthcare resources than those who are not unwell (Stein 2006; Baune 2007). Even when attendance is high, quality of care can be low (Jones 2005; Salsberry 2005). Therefore predictors of adequate care should include measures of both quality and quantity, and deficits in either may result in failing care. Clinicians' low confidence or knowledge regarding mental-physical comorbidity may also compromise medical care for this vulnerable population.

\section{Inequalities in surgical care}

Having a diagnosis of schizophrenia is linked to poor surgical outcomes. In a review of appendectomies in people with schizophrenia in the USA, Cooke et al (2007) found that they presented late in the natural course of the illness $(80 \%$ with advanced appendicitis, perforated or gangrenous at the time of surgery). Morbidity and mortality rates were much higher than in the control group. A systematic review and meta-analysis has confirmed that schizophrenia is associated with an increased rate of fractures (Stubbs 2015). In a US survey, SMI was also associated with higher odds of inhospital post-operative complications following total hip or knee arthroplasty, with the rate of adverse events being most highly associated with a diagnosis of schizophrenia (Buller 2015). Similarly, two large studies from Taiwan and Japan showed significantly higher postoperative adverse outcomes, possibly with increased mortality, in people with schizophrenia when compared with those without mental disorders (Liao 2013; Maeda 2014).

\section{Inequalities in assessing and monitoring comorbid physical ill health}

Although there are many reasons for inequalities in mortality and morbidity, those relating to the attitudes and behaviour of clinicians are most concerning. For example, negative attitudes towards people with mental illness held by healthcare professionals may mean that some physical symptoms are wrongly ignored or are attributed to mental disorder (Thornicroft 2007). In a survey of nearly 800 people living with schizophrenia in 27 European countries, $17 \%$ felt that they experienced discrimination when treated for physical health problems (Harangozo 2013). Similarly, a North American survey of 250 people with schizophrenia reported that $49 \%$ believed that doctors took their medical problems less seriously after discovering that they had a psychiatric diagnosis (National Alliance on Mental Illness 2008). A national survey in primary care in England showed that the percentage of patients who received the identical medical checks (body mass index, blood pressure, blood glucose and cholesterol) in the previous 15 months was much higher among patients with diabetes than those with SMI $(97.3 \%$ v. 74.7\%, $P<0.001$ ) (Mitchell 2013b).

To improve this situation, numerous national and international guidelines have been published for the monitoring of physical health in people with mental illness (De Hert 2011). However, a recent review showed considerable variation in the level of detail, methodology and recommendations (such as timing of monitoring and proposed therapeutic interventions) (Mitchell 2012b). In the UK, the National Institute for Health and Care Excellence (NICE) recommends annual monitoring of physical health for people with established psychosis (National Collaborating Centre for Mental Health 2014). NICE recommends more frequent monitoring by mental health services in the first year of a psychotic illness, with the responsibility subsequently transferred to primary care under 
shared-care arrangements. Numerous studies from the field of early intervention suggest that early physical health intervention and health promotion for patients with mental illness are likely to be the most important type of intervention (Mitchell 2015a). The good news is that screening for physical comorbidity in SMI has indeed improved, but the bad news is that (with exceptions) it is still generally deficient (Baller 2015). For example, in the UK National Audit of Schizophrenia, a 12-month retrospective review was performed of the case records of 5091 people with a diagnosis of schizophrenia or schizoaffective disorder attending community mental health services in England and Wales. Data were collected on nine key aspects of physical health. Physical health monitoring was poor, with less than $25 \%$ of patients having all nine parameters documented; and where problems were identified, treatment was rarely offered (Crawford 2014).

\section{Failure to address metabolic complications in psychiatric care}

Data from the National Ambulatory Medical Care Survey from 1992 to 1996 in the USA found that, in 1600 office visits of patients with mental illness who were documented smokers, psychiatrists offered smoking-cessation counselling at $12 \%$ of the visits, and diet and exercise counselling at $6 \%$ and $4 \%$ of visits respectively (Himelhoch 2003). A more recent meta-analysis of comparative studies found equivalent rates of smoking-cessation advice given to medical $v$. psychiatric patients (Mitchell 2015b). This may appear to be an improvement, but as smoking is approximately twice as common in patients with mental illness the level of advice for this group should also be double.

It is clear that, if inequalities are to be addressed, clinicians must respond to physical health problems in patients with mental illness when they are discovered.Unfortunately, this often does not happen. In the largest controlled study of its type, Clinical Antipsychotic Trials of Intervention Effectiveness (CATIE), $88 \%$ of patients with dyslipidaemia were untreated, as were $62 \%$ with hypertension and $38 \%$ with diabetes (Nasrallah 2006). Correll et al (2008) found that $62 \%$ of patients treated with second-generation (atypical) antipsychotics who had elevated low-density lipoprotein (LDL) levels did not receive medical treatment, despite the fact that they were in-patients. Bernardo et al (2009) found that among in-patients with schizophrenia, only $60 \%$ of those with diabetes, $28 \%$ of those with hypertension and $14 \%$ of those with dyslipidaemia received active medical treatment. Several sources of evidence suggest that psychiatrists are slow to change drugs linked with metabolic syndrome even after complications occur (Hermes 2013). For example, in a multi-centre study in Denmark and Sweden, in consecutively screened in- and outpatients with schizophrenia spectrum disorders continuously treated for at least 3 months with atypical antipsychotics, the high rate of metabolic syndrome did not elicit much decisive action on the part of the treating psychiatrists (Larsen 2011). The most frequent action taken was dietary and exercise advice (for $75 \%$ of patients), while a laboratory or blood pressure follow-up was advised for $54 \%$ and $19 \%$ of patients respectively. Change of antipsychotic was recommended for only $10 \%$ of patients, and no action was taken for a further $11 \%$.

\section{Improving parity of esteem}

Improving the physical health of patients with SMI

Population-based initiatives are promising but rare, although there is some evidence that fluoridated water reduces inequality in dental care for people with SMI (Kisely 2011). An initial Cochrane review in 2010 was unable to find a single randomised controlled trial reporting evidence on the effectiveness or cost-effectiveness of guidelines advising on the monitoring of physical health in people with severe mental health problems (Tosh 2010). Since then there have been several randomised trials on programmes to prevent physical ill health in people with mental disorders, largely by addressing risk factors. These risk factors tend to exacerbate with time if not addressed (Heald 2016). Interventions have focused on smoking cessation, weight management, glucose control or broader lifestyle intervention in depression/ anxiety and SMI (Barnett 2008; Park 2013; Bruins 2014; Vancampfort 2016; Taylor 2017). One recent meta-analysis (Park 2013) found that the drop-out rate was modest and a second found that overall, lifestyle interventions were effective in both weight loss and prevention of weight gain (Vancampfort 2015). Bruins et al (2014) identified 10 studies reporting on cardiometabolic risk factors, and these showed that lifestyle interventions led to significant improvements in waist circumference, triglycerides, fasting glucose and insulin, but not blood pressure and cholesterol. Two trials (Bartels 2013; Green 2015) found different outcomes. The In SHAPE trial focused on exercise (12 months of weekly sessions with a fitness trainer plus gym membership) (Bartels 2013). The prospective primary outcome was weight loss and the secondary outcomes were fitness, blood pressure, lipids and programme adherence. There was a clinically significant reduction in cardiovascular risk in $49 \%$ of participants and improvements in fitness and diet, but not in weight. In the STRIDE trial, 


\begin{tabular}{|c|c|}
\hline \multicolumn{2}{|c|}{ Ten priority areas for improvement } \\
\hline Prevention/public health & $\begin{array}{l}1 \text { Incorporating mental health into public health programmes } \\
2 \text { Health promotion and prevention among people with severe } \\
\text { mental illness }\end{array}$ \\
\hline General practice & $\begin{array}{l}3 \text { Improving management of 'medically unexplained symptoms' in } \\
\text { primary care } \\
4 \text { Strengthening primary care for the physical health needs of } \\
\text { people with severe mental illness }\end{array}$ \\
\hline Chronic disease management & $\begin{array}{l}5 \text { Supporting the mental health of people with long-term conditions } \\
6 \text { Supporting the mental health and well-being of carers }\end{array}$ \\
\hline Hospital care & $\begin{array}{l}7 \text { Mental health in acute general hospitals } \\
8 \text { Physical health in mental health in-patient facilities }\end{array}$ \\
\hline Community/social care & $\begin{array}{l}9 \text { Integrated support for perinatal mental health } \\
10 \text { Supporting the mental health needs of people in residential homes }\end{array}$ \\
\hline
\end{tabular}

FIG 1

Ten priority areas for improvement in mental healthcare (from Naylor et al (2016), redrawn with permission of The King's Fund).

The role of psychiatrists in educating Foundation Programme doctors in the UK was discussed in the March 2017 issue of BJPsych Advances: see Perry et al,

'Psychiatry in the Foundation Programme: an overview for supervisors' (pp. 123-130) and Sarkar et al, 'Going beyond "good enough" teaching in psychiatric training (pp. 131-142). Ed. participants attended weekly groups providing moderate exercise and dietary advice and achieved an average $4.4 \mathrm{~kg}$ weight loss over 6 months, but gained some of this weight back during the maintenance phase (Green 2015). More recently, the CHANGE trial failed to find a benefit of lifestyle coaching on 10-year risk of cardiovascular disease in patients with schizophrenia spectrum disorders and abdominal obesity (Speyer 2016).

Given the heterogeneity in outcomes, lessons from lifestyle intervention for other conditions, such as diabetes, may be warranted. For example, successful programmes tend to have multiple strategies (nutrition and physical activity), faceto-face contact, longer duration, longer follow-up and trained treatment providers (Ward 2015). However, the biggest lesson is that the later we leave intervention for physical comorbidities such as obesity, the poorer the outcomes. The gold standard is prevention (Moore 2015; Mitchell 2015a). Therefore, the challenge is to recognise that all patients with SMI, and many with other mental health conditions, are at risk of physical complications and all such patients need a great deal of help and support to avoid weight gain, smoking, inactivity and frank metabolic conditions such as diabetes. Tools have been developed to assist healthcare professionals, for example, the Lester UK Adaptation of the Positive Cardiometabolic Health Resource (produced through collaboration between the Royal Colleges of General Practitioners and Psychiatrists and supported by the National Audit of Schizophrenia). This resource has been trialed in the Advancing Quality Alliance (AQuA) 'Don't Just Screen - Intervene' initiative across five early-onset psychosis services and it increased cardiometabolic risk-screening from 10 to $63 \%$ (Greenwood 2016). Specific resources to assist mental health nurses
(White 2009) and general practice nurses (Hardy 2015a) have also been available in the UK for a number of years.

\section{Integrating mental and physical healthcare services}

A recent King's Fund report (Naylor 2016) emphasised the need to bring together physical and mental healthcare, rather than bridging the gaps between health and social care or between primary and secondary care. It identified ten priority areas for improvement (Fig. 1) and encouraged this through the following:

- vanguard sites led by NHS England, with integrated approaches to physical and mental health

- all health and social care professionals have a part to play in taking a ‘whole-person' approach to physical and mental health

- medical Royal Colleges should redesign curricula so that all health professionals have a common foundation in mental as well as physical health ${ }^{\dagger}$

- local authorities should assess how mental health and well-being interact with other public health priorities

- commissioners should take advantage of new payment systems and contracting approaches to overcome some of the current barriers to integration

- mental health trusts should consider having a board-level champion for physical health, and vice versa in acute trusts.

\section{Encouraging health-seeking behaviour}

A Healthwatch briefing published in February 2016 stated that 77 out of the 152 local Healthwatch groups reported that mental health was the most important health and social care priority to their local people (Healthwatch England 2016). Its authors suggested that engaging individuals in their own care makes a real difference in enabling mental health services to deliver for people.

Help-seeking is influenced by stigma. There have been attempts to address inequalities by initiatives to reduce stigma in the general population, with the expectation that this would influence help-seeking and clinicians' attitudes (Time to Change 2008). However, there is limited evidence that stigma interventions are effective in reducing perceived or self-stigma (Griffiths 2014); this suggests that there is a need for healthcare services to take a much more proactive approach in encouraging and assisting people with mental illness to access appropriate care and treatment. Recognising this, the British Medical Association (BMA) has developed pragmatic recommendations in three areas: mortality, prevention and early intervention, and training and workforce planning (Box 2). 


\section{Addressing and improving the mortality gap}

Addressing the mortality gap requires a comprehensive series of changes rather than change in one particular disparity. The BMA recommends that a national mortality review system be implemented across the UK (Box 2). This would make information available to healthcare providers about the circumstances of deaths and the care provided in the period leading up to a death. Similarly, the Mental Health Taskforce recommends that NHS England should define a quantified national reduction in premature mortality among people with SMI, and an operational plan to begin achieving it from 2017/2018 (Mental Health Taskforce 2016). Failures in care or systems could then be addressed to prevent deaths and improve services in the future.

The key reversible areas that might reduce the mortality gap include addressing unhelpful health behaviours (especially smoking), reducing metabolic risks (e.g. obesity, inactivity) before and after starting psychiatric treatment, prompt recognition and treatment of medical comorbidities and improving quality of medical care for patients with known mental illness. Unfortunately, the evidence that specific interventions reduce excess mortality in those with mental ill health is currently sparse (Baxter 2016). Two Cochrane reviews on health outcomes in people with SMI allocated to integrative community care management or intensive case management found no effect on mortality, and seven studies of lifestyle intervention in SMI found no effect on all-cause mortality (see Baxter 2016).

\section{Promoting prevention and early intervention strategies}

The BMA states that integration of intellectual disability, mental and physical healthcare should be improved (Box 2). Despite this, reform has been slow, with little evidence that inequalities are reducing (Brekke 2013). Models of integrated care include embedding of general practitioners (GPs) or hospital specialists within mental health programmes, housing medical and mental health services together, and collaborative care between independent physical and mental healthcare providers. A number of studies involving collaborative care to address risk factors for poor health in schizophrenia have reported improvement of physical and mental quality of life (Woltmann 2012), but only two (the PCARE study and the SMAHRT study) have attempted to improve medical care directly. In the PCARE study (Druss 2010), the intervention group received an average of $59 \%$ of recommended preventive services, compared with $22 \%$ in the usual-care group. They also received a significantly higher proportion of evidence-based

services for cardiometabolic conditions $(35 \% \mathrm{v}$. $28 \%$ ) and were more likely to receive a physical examination. In the SMAHRT study (Kilbourne 2013), patients receiving the intervention had reduced systolic and diastolic blood pressure, as well as reduced manic symptoms. However, when collaborative care is implemented in practice, there are limited changes to processes of care, as physical health and mental health professionals often maintain their physical and mental health role boundaries (Wells 2000; Knowles 2013). In general, there is often a cultural divide between researchers, practitioners and administrators which can prevent research becoming standard clinical practice (Eccles 2005).

\section{BOX 2 Summary of the British Medical Association's parity of esteem recommendations}

\section{Mortality}

- A national mortality review system should be implemented across the UK

Promoting prevention and early intervention strategies

- The integration of intellectual disability, mental and physical healthcare should be improved

- The wider application of the collaborative care model should be explored

- To strengthen collaborative care, generalists in and out of hospitals should work closely with a multidisciplinary team to support patients with co- or multimorbidities in a more integrated way

- A liaison psychiatry service (including paediatric, intellectual disability and older adult liaison) should be made available in all hospitals

- Mental health trusts should appoint a liaison physician (in psychiatric wards) to support the physical health needs of hospital in-patients with mental health problems and intellectual disabilities

- Health professionals with responsibilities for commissioning and/or public health should ensure that they place equal value on mental and physical health when setting their commissioning priorities

- Mental health should be integrated into all aspects of the work of commissioners and public health directors

- Commissioners must have accurate, accessible information on the physical, mental and intellectual disability needs of the population that they are serving
- It is strongly recommended that commissioning bodies should ensure that at least one commissioner is a mental health and/ or intellectual disability professional

- Healthcare providers must ensure that they are compliant with their duties under the Equality Act 2010

- Providers' compliance with legislation and guidelines should be monitored by healthcare regulators and inspectors such as Monitor and Healthcare Improvement Scotland

\section{Training and workforce planning}

- Undergraduate and postgraduate medical training should shift from teaching mental health separately in a 'module' format to integrating mental health and intellectual disability into the core curriculum

- There should be adequate workforce planning to ensure that all Foundation Programme doctors and general practitioners (GPs) in training have a placement in psychiatry, liaison psychiatry or intellectual disability - this is an area for action for the Royal College of Psychiatrists and the Royal College of General Practitioners

- All doctors should understand diagnostic overshadowing, comorbidity problems and the requirements of the Mental Capacity Act 2005 and the Equality Act 2010

- All healthcare professionals should undertake mental health and intellectual disability awareness training as part of their continuing professional development (Adapted from: BMA Science and Education Department, BMA Board of Science 2014) 
The BMA recommends that commissioners place equal value on mental and physical health when setting their commissioning priorities (Box 2), yet financial incentives for prevention in primary care within the Quality and Outcomes Framework (QOF) such as screening for depression and measuring all cardiovascular disease risk factors in people with SMI have been removed in both England and Wales (Graham 2014). As the contacts that these patients have with GPs have reduced significantly over the past 20 years - from 13 a year (Nazareth 1992) to just 3 a year (Reilly 2012) - and they have little contact with practice nurses (Wells 2000), there is not much chance to provide opportunistic screening. In psychiatric hospitals there is substantial variation in quality of care, for example, in care received by patients with schizophrenia (Jørgensen 2015). This is despite the CQUIN described earlier and the option of local CQUINs. Financial incentive schemes in healthcare can lead to improvements in the quality of care for rewarded activities, but they can also lead to neglect of the non-incentivised aspects of patient care (Doran 2011). Additionally, the targets of these incentives can be met and guidance can be followed without any real commitment from the organisation to making sustainable changes that will improve practice.

Overall funding for mental healthcare has suffered. Total investment in mental health services across England decreased in real terms by 1\% between 2010-2011 and 2011-2012, and by 2.3\% between 2011-2012 and 2013-2014, despite a $13.3 \%$ increase in the average number of referrals being handled by community mental health teams (Mental Health Strategies 2012; Buchanan 2013). A report by the Mental Health Taskforce (2016) stated that, in 2013-2014, the NHS spent £9.2 billion on mental health services - less than a tenth of its budget - even though one in four people experience a mental health problem each year. The report announced that by 2020, new funding should increase access to evidence-based psychological therapies for conditions such as anxiety, depression and stress and to a screening programme to ensure that more people with severe mental health problems get help for physical health conditions such as heart disease and diabetes (as required by the Equality Act 2010). The chief executive of the mental health charity Mind, who led the taskforce, was reported to have said that, although the extra funding was crucial, some stigma around mental illness 'still prevails, right the way inside the health service, as it does in society, and this needs to change' ('Triggle 2016). There is evidence to suggest that the negative attitudes of healthcare professionals towards people with a mental illness are detrimental to recovery and reduce their use of healthcare services (Horsfall 2010). Improving the attitudes of healthcare professionals looking after people with mental illness could have a major positive impact on the health of this group. Research has shown that educational interventions can be effective in decreasing stigmatising attitudes among healthcare professionals, especially those in general healthcare who have little or no formal mental health training (Henderson 2014).

\section{Training and workforce planning}

The Mental Health Taskforce's report for the NHS in England suggests that Health Education England (HEE) should work with NHS England, Public Health England (PHE), professional bodies, charities, experts-by-experience and others to develop a costed multidisciplinary workforce strategy for the future shape and skills mix of the workforce that addresses training needs for both new and existing NHS-funded staff (Mental Health Taskforce 2016). It recommends that core training should include basic mental health awareness, mental health law, public mental health, compassion and communication skills, common physical health problems, shared decision-making, prevention of mental illness, empowering people to understand their own strengths and to use self-help strategies, and promote carer involvement as well as information sharing.

So far, educational initiatives funded by HEE have been regional and they have not been rolled out nationally. One example is the practice nurse project, which was led by one of us (S.H.) and funded by Health Education North Central East London (HENCEL) (Hardy 2015b). This 10-module training developed for practice nurses covers most areas recommended by HEE and was successfully delivered by mental health nurses working in the practice nurses' localities. Although frequently cited as an example of good practice, there has been no vehicle to disseminate it countrywide. S.H. now works one day a week with a small charity (the Charlie Waller Memorial Trust) and through this offers the course for identified trainers free of charge. She has found that organisations are very interested, but appear to struggle with setting up their own training plan. The problems are mainly due to the inability to release nurses from practice both to receive training and to train others. This suggests the need to educate commissioners as well as clinicians to enable learning to take place. A national plan as recommended by the taskforce could resolve some of these problems. Once staff have received training, organisations need to enable them to teach their peers and put their learning into practice. 


\section{Conclusions}

Parity of esteem continues to be a major concern. Five years after the government's mental health strategy 'No Health without Mental Health' (the aim of which was to improve outcomes, physical health and experience of care for people with mental health problems and to reduce avoidable harm and stigma), a new report appeared highlighting deficits and making recommendations (Mental Health Taskforce 2016). This shows continuing inadequate provision and worsening outcomes, suggesting that policies on their own change neither practice nor provision. All clinicians need to be aware of parity of esteem, as do all commissioners. Careful planning and organisation are required so that extra funding is used to reduce inequalities in care and ultimately equalise parity of esteem between physical and mental health.

\section{References}

Baller JB, McGinty EE, Azrin ST, et al (2015) Screening for cardiovascular risk factors in adults with serious mental illness: a review of the evidence. BMC Psychiatry, 15: 55

Barnett PG, Wong W, Hall S (2008) The cost-effectiveness of a smoking cessation program for out-patients in treatment for depression. Addiction, 103: 834-40.

Bartels SJ, Pratt SI, Aschbrenner KA, et al (2013) Clinically significant improved fitness and weight loss among overweight persons with serious mental illness. Psychiatric Services, 64: 729-36.

Baune BT, Adrian I, Jacobi F (2007) Medical disorders affect health outcome and general functioning depending on comorbid major depression in the general population. Journal of Psychosomatic Research, 62: 109-18.

Baxter AJ, Harris MG, Khatib Y, et al (2016) Reducing excess mortality due to chronic disease in people with severe mental illness: meta-review of health interventions. British Journal of Psychiatry, 208: 322-9

Bernardo M, Cañas F, Banegas JR, et al (2009) Prevalence and awareness of cardiovascular risk factors in patients with schizophrenia: a crosssectional study in a low cardiovascular disease risk geographical area. European Psychiatry, 24: 431-41

BMA Science and Education Department, BMA Board of Science (2014) Recognising the Importance of Physical Health in Mental Health and Intellectual Disability: Achieving Parity of Outcomes. British Medical Association.

Brekke J, Siantz E, Pahwa R, et al (2013) Reducing health disparities for people with serious mental illness: development and feasibility of a peer health navigation intervention. Best Practices in Mental Health, 9: 62-82.

Brown S, Kim M, Mitchell C, et al (2010) Twenty-five year mortality of a community cohort with schizophrenia. British Journal of Psychiatry, 196: $116-21$

Bruins J, Jörg F, Bruggeman R, et al (2014) The effects of lifestyle interventions on (long-term) weight management, cardiometabolic risk and depressive symptoms in people with psychotic disorders: a meta-analysis. PLOS ONE, 9(12): e112276.

Buchanan M (2013) Funds cut for mental health trusts in England. BBC News, 12 December (http://www.bbc.co.uk/news/health-25331644). Accessed 8 November 2016.

Buller LT, Best J, Klika AK, et al (2015) The influence of psychiatric comorbidity on perioperative outcomes following primary total hip and knee arthroplasty; a 17-year analysis of the national hospital discharge survey database. Journal of Arthroplasty, 30: 165-70.

Clement S, Schauman 0, Graham T, et al (2015) What is the impact of mental health-related stigma on help-seeking? A systematic review of quantitative and qualitative studies. Psychological Medicine, 45: 11-27.
Cooke BK, Magas LT, Virgo KS, et al (2007) Appendectomy for appendicitis in patients with schizophrenia. American Journal of Surgery, 193: 41-8.

Correll CU, Kane JM, Manu P (2008) Identification of high-risk coronary heart disease patients receiving atypical antipsychotics: single lowdensity lipoprotein cholesterol threshold or complex national standard? Journal of Clinical Psychiatry, 69: 578-3.

Crawford MJ, Jayakumar S, Lemmey SJ, et al (2014) Assessment and treatment of physical health problems among people with schizophrenia: national cross-sectional study. British Journal of Psychiatry, 205: 473-7.

De Hert M, Vancampfort D, Correll CU, et al (2011) Guidelines for screening and monitoring of cardiometabolic risk in schizophrenia: systematic evaluation. British Journal of Psychiatry, 199: 99-105.

Doran T, Kontopantelis E, Valderas J, et al (2011) Effect of financial incentives on incentivised and non-incentivised clinical activities: longitudinal analysis of data from the UK Quality and Outcomes Framework. BMJ, 342: d3590.

Druss BG, von Esenwein SA, Compton MT, et al (2010) A randomized trial of medical care management for community mental health settings: the Primary Care Access, Referral, and Evaluation (PCARE) study. American Journal of Psychiatry, 167: 151-9.

Druss BG, Bradford D, Rosenheck RA, et al (2011) Quality of medical care and excess mortality in older patients with mental disorders. Archives of General Psychiatry, 58: 565-72.

Eccles M, Grimshaw J, Walker A, et al (2005) Changing the behavior of healthcare professionals: the use of theory in promoting the uptake of research findings. Journal of Clinical Epidemiology, 58: 107-12.

Fleischhacker WW, Cetkovich-Bakmas M, De Hert M, et al (2008) Comorbid somatic illnesses in patients with severe mental disorders: clinical, policy, and research challenges. Journal of Clinical Psychiatry, 69: $514-9$

Graham CC, Chitnis AJ, Turner P, et al (2014) Why all GPs should be bothered about Billy. British Journal of General Practice, 64: 15

Green CA, Yarborough BJ, Leo MC, et al (2015) The STRIDE weight loss and lifestyle intervention for individuals taking antipsychotic medications: a randomized trial. American Journal of Psychiatry, 172: 71-81.

Greenwood PJ, Shiers DE (2016) Don't just screen, intervene: a quality improvement initiative to improve physical health screening of young people experiencing severe mental illness. Mental Health Review Journal, 21: 48-60.

Griffiths KM, Carron-Arthur B, Parsons A, et al (2014) Effectiveness of programs for reducing the stigma associated with mental disorders: a meta-analysis of randomized controlled trials. World Psychiatry, 13: $161-75$.

Harangozo J, Reneses B, Brohan E, et al (2013) Stigma and discrimination against people with schizophrenia related to medical services. International Journal of Social Psychiatry, 60: 359-66.

Hardy S (2015a) The perplexing issue of severe mental illness, physical health screening and primary care in England. Journal of Psychiatric and Mental Health Nursing, 22: 647-52.

Hardy S, Kingsnorth R (2015b) Mental health nurses can increase capability and capacity in primary care by educating practice nurses: an evaluation of an education programme in England. Journal of Psychiatric and Mental Health Nursing, 22: 270-7.

Heald AH, Martin JL, Payton T, et al (2016) Changes in metabolic parameters in patients with severe mental illness over a 10-year period: a retrospective cohort study. Australian and New Zealand Journal of Psychiatry, 14 Jan: 0004867415625817 [Epub ahead of print].

Healthwatch England (2016) Mental Health: The Public's Number One Issue for 2016. Healthwatch.

Henderson C, Noblett J, Parke H, et al (2014) Mental health-related stigma in health care and mental health-care settings. Lancet Psychiatry, 1: 467-82.

Hermes ED, Sernyak MJ, Rosenheck RA (2013) Prescription of secondgeneration antipsychotics: responding to treatment risk in real-world practice. Psychiatric Services, 64: 238-44.

Himelhoch S, Daumit G (2003) To whom do psychiatrists offer smokingcessation counseling? American Journal of Psychiatry, 160: 2228-30. 


MCQ answers
$1 d \quad 2 a$
$1 d$

Horsfall J, Cleary M, Hunt G (2010) Stigma in mental health: clients and professionals. Issues in Mental Health Nursing, 31: 450-5.

Jones LE, Carney CP (2005) Mental disorders and revascularization procedures in a commercially insured sample. Psychosomatic Medicine, 67: $568-76$.

Jørgensen M, Mainz J, Svendsen ML, et al (2015) Improving quality of care among patients hospitalised with schizophrenia: a nationwide initiative. British Journal of Psychiatry Open, 1: 48-53.

Kessler RC, Berglund PA, Bruce ML, et al (2001) The prevalence and correlates of untreated serious mental illness. Health Services Research, 36: 987-1007.

Kilbourne AM, Goodrich DE, Lai Z, et al (2013) Randomized controlled trial to assess reduction of cardiovascular disease risk in patients with bipolar disorder: the Self-Management Addressing Heart Risk Trial (SMAHRT). Journal of Clinical Psychiatry, 74: e655-62.

Kisely S, Quek L-H, Pais J, et al (2011) Advanced dental disease in people with severe mental illness: systematic review and meta-analysis. British Journal of Psychiatry, 199: 187-93.

Knowles S, Chew-Graham C, Coupe N, et al (2013) Better together? A naturalistic qualitative study of inter-professional working in collaborative care for co-morbid depression and physical health problems. Implementation Science, 8: 110

Larsen JT, Fagerquist M, Holdrup M, et al (2011) Metabolic syndrome and psychiatrists' choice of follow-up interventions in patients treated with atypical antipsychotics in Denmark and Sweden. Nordic Journal of Psychiatry, 65: 40-6.

Lawrence D, Kisely S (2010) Inequalities in healthcare provision for people with severe mental illness. Journal of Psychopharmacology, 24: 61-8.

Liao CC, Shen WW, Chang CC, et al (2013) Surgical adverse outcomes in patients with schizophrenia: a population-based study. Annals of Surgery, 257: 433-8.

Lord 0, Malone D, Mitchell AJ (2010) Receipt of preventive medical care and medical screening for patients with mental illness: a comparative analysis. General Hospital Psychiatry, 32: 519-43.

Maeda T, Babazono A, Nishi T, et al (2014) Influence of psychiatric disorders on surgical outcomes and care resource use in Japan. General Hospital Psychiatry, 36: 523-7.

McCreadie RG, Stevens H, Henderson J, et al (2004) The dental health of people with schizophrenia. Acta Psychiatrica Scandinavica, 110: 306-10.

Mental Health Strategies (2012) 2011/12 National Survey of Investment in Adult Mental Health Services. Department of Health.

Mental Health Taskforce (2016) The Five Year Forward View for Mental Health. Mental Health Taskforce to the NHS in England.

Mitchell AJ, Malone D, Doebbeling CC (2009) Quality of medical care for people with and without comorbid mental illness and substance misuse: systematic review of comparative studies. British Journal of Psychiatry, 194: $491-9$.

Mitchell AJ, Lord 0 (2010) Do deficits in cardiac care influence high mortality rates in schizophrenia? A systematic review and pooled analysis. Journal of Psychopharmacology, 24: 69-80.

Mitchell AJ, Lord 0, Malone D (2012a) Differences in the prescribing of medication for physical disorders in individuals with $v$. without mental illness: meta-analysis. British Journal of Psychiatry, 201: 435-43.

Mitchell AJ, Delaffon V, Vancampfort D, et al (2012b) Guideline concordant monitoring of metabolic risk in people treated with antipsychotic medication: systematic review and meta-analysis of screening practices. Psychological Medicine, 42: 125-47.

Mitchell AJ, Vancampfort D, Sweers K, et al (2013a) Prevalence of metabolic syndrome and metabolic abnormalities in schizophrenia and related disorders: a systematic review and meta-analysis. Schizophrenia Bulletin, 39: 306-18.

Mitchell AJ, Hardy SA (2013b) Screening for metabolic risk among patients with severe mental illness and diabetes: a national comparison. Psychiatric Services, 64: 1060-3.
Mitchell AJ, Pereira IE, Yadegarfar M, et al (2014) Breast cancer screening in women with mental illness: comparative meta-analysis of mammography uptake. British Journal of Psychiatry, 205: 428-35.

Mitchell AJ, De Hert M (2015a) Promotion of physical health in persons with schizophrenia: can we prevent cardiometabolic problems before they begin? Acta Psychiatrica Scandinavica, 132: 83-5.

Mitchell AJ, Vancampfort D, De Hert M, et al (2015b) Do people with mental illness receive adequate smoking cessation advice? A systematic review and meta-analysis. General Hospital Psychiatry, 37: 14-23.

Moore S, Shiers D, Daly B, et al (2015) Promoting physical health for people with schizophrenia by reducing disparities in medical and dental care. Acta Psychiatrica Scandinavica, 132: 109-21.

Mujica-Mota RE, Roberts M, Abel G, et al (2015) Common patterns of morbidity and multi-morbidity and their impact on health-related quality of life: evidence from a national survey. Quality of Life Research, 24: 909-18.

Nasrallah HA, Meyer JM, Goff DC, et al (2006) Low rates of treatment for hypertension, dyslipidemia and diabetes in schizophrenia: data from the CATIE schizophrenia trial sample at baseline. Schizophrenia Research, 86: 15-22.

National Alliance on Mental IIIness (2008) Schizophrenia: Public Attitudes, Personal Needs: Views from People Living with Schizophrenia, Caregivers, and the General Public. NAMI.

Naylor C, Das P, Ross S, et al (2016) Bringing together Physical and Mental Health: A New Frontier for Integrated Care. King's Fund.

Nazareth ID, King MB (1992) Controlled evaluation of management of schizophrenia in one general practice: a pilot study. Family Practitioner, 9: 171-2.

National Collaborating Centre for Mental Health (2014) Psychosis and Schizophrenia in Adults: Treatment and Management (NICE Clinical Guideline 178). NICE.

NHS England (2014) A Call to Action: Achieving Parity of Esteem; Transformative Ideas for Commissioners. NHS England.

Olfson M, Gerhard T, Huang C, et al (2015) Premature mortality among adults with schizophrenia in the United States. JAMA Psychiatry, 72: 1172-81.

Paddison CAM, Saunders CL, Abel GA, et al (2015) Why do patients with multimorbidity in England report worse experiences in primary care? Evidence from the General Practice Patient Survey. BMJ Open, 5: e006172.

Park A-L, McDaid D, Weiser P, et al (2013) Examining the cost effectiveness of interventions to promote the physical health of people with mental health problems: a systematic review. BMC Public Health, 13: 787.

Reilly S, Planner C, Hann M, et al (2012) The role of primary care in service provision for people with severe mental illness in the United Kingdom. PLOS ONE, 7(5): e36468.

Royal College of Psychiatrists (2013) Whole-Person Care: From Rhetoric to Reality. Achieving Parity between Mental and Physical Health (Occasional Paper OP88). Royal College of Psychiatrists.

Salsberry PJ, Chipps E, Kennedy C (2005) Use of general medical services among Medicaid patients with severe and persistent mental illness. Psychiatric Services, 56: 458-62.

Smith DJ, Langan J, McLean G, et al (2013) Schizophrenia is associated with excess multiple physical-health comorbidities but low levels of recorded cardiovascular disease in primary care: cross-sectional study. BMJ Open, 3: e002808.

Speyer H, Brix Nørgaard HC, Birk M, et al (2016) The CHANGE trial: no superiority of lifestyle coaching plus care coordination plus treatment as usual compared to treatment as usual alone in reducing risk of cardiovascular disease in adults with schizophrenia spectrum disorders and abdominal obesity. World Psychiatry, 15: 155-65.

Stein MB, Cox BJ, Afifi T0, et al (2006) Does co-morbid depressive illness magnify the impact of chronic physical illness? A population-based perspective. Psychological Medicine, 36: 587-96.

Stubbs B, Gaughran F, Mitchell AJ, et al (2015) Schizophrenia and the risk of fractures: a systematic review and comparative meta-analysis. General Hospital Psychiatry, 37: 126-33. 
Taylor J, Stubbs B, Hewitt C, et al (2017) The effectiveness of pharmacological and non-pharmacological interventions for improving glycaemic control in adults with severe mental illness: a systematic review and meta-analysis. PLOS ONE, 12(1): e0168549. doi:10.1371/journal. pone.0168549

Thornicroft G, Rose D, Kassam A (2007) Discrimination in health care against people with mental illness. International Review of Psychiatry, 19: 113-22.

Time to Change (2008) Mental health and stigma. Mind and Rethink Mental IIIness (http://www.time-to-change.org.uk/mental-healthstigma). Accessed 10 November 2016.

Torniainen M, Mittendorfer-Rutz E, Tanskanen A, et al (2015) Antipsychotic treatment and mortality in schizophrenia. Schizophrenia Bulletin, 41: 656-63.

Tosh G, Clifton A, Mala S, et al (2010) Physical health care monitoring for people with serious mental illness. Cochrane Database of Systematic Reviews, 3: CD008298.

Triggle N (2016) Nation must focus on mental health - PM. BBC News, 15 February (http://www.bbc.co.uk/news/health-35565216). Accessed 8 November 2016.

Vancampfort D, Rosenbaum S, Schuch FB, et al (2015) Prevalence and predictors of treatment dropout from physical activity interventions in schizophrenia: a meta-analysis. General Hospital Psychiatry, 39: 15-23.

Vancampfort D, Rosenbaum S, Schuch FB, et al (2016) Prevalence and predictors of treatment dropout from physical activity interventions in schizophrenia: a meta-analysis. General Hospital Psychiatry, 39: 15-23.

Vigo D, Thornicroft G, Atun R (2016) Estimating the true global burden of mental illness. Lancet Psychiatry, 3: 171-8.

Walker ER, McGee RE, Druss BG (2015) Mortality in mental disorders and global disease burden implications: a systematic review and metaanalysis. JAMA Psychiatry, 72: 334-41.
Ward MC, White DT, Druss BG (2015) A meta-review of lifestyle interventions for cardiovascular risk factors in the general medical population: lessons for individuals with serious mental illness. Journal of Clinical Psychiatry, 76: e477-86

Wells K, Sherbourne C, Schoenbaum M, et al (2000) Impact of disseminating quality improvement programs for depression in managed primary care. JAMA, 283: 212-20.

Wey MC, Loh S, Doss JG, et al (2016) The oral health of people with chronic schizophrenia: a neglected public health burden. Australian and New Zealand Journal of Psychiatry, 50: 685-94.

White J, Gray R, Jones M (2009) The development of the serious mental illness physical Health Improvement Profile. Journal of Psychiatric and Mental Health Nursing, 16: 493-8.

Whiteford HA, Degenhardt L, Rehm J, et al (2013) Global burden of disease attributable to mental and substance use disorders: findings from the Global Burden of Disease Study 2010. Lancet, 382: 1575-86.

Whiteford HA, Ferrari AJ, Degenhardt L, et al (2015) The global burden of mental, neurological and substance use disorders: an analysis from the Global Burden of Disease Study 2010. PLOS ONE, 10(2): e0116820.

Woltmann E, Grogan-Kaylor A, Perron B, et al (2012) Comparative effectiveness of collaborative chronic care models for mental health conditions across primary, specialty, and behavioral health care settings: systematic review and meta-analysis. American Journal of Psychiatry, 169: 790-804.

Woodhead C, Ashworth M, Broadbent M, et al (2016) Cardiovascular disease treatment among patients with severe mental illness: a data linkage study between primary and secondary care. British Journal of General Practice, 66: e374-81.

World Health Organization (2013) Mental Health Atlas - 2011 Country Profiles. WHO (http://www.who.int/mental_health/evidence/atlas/ profiles).

\section{MCOs}

Select the single best option for each question stem

1 The increased mortality among people with current or past mental disorders:

a is mostly attributable to suicide

$\mathrm{b}$ is exclusively due to the psychotropic drugs that contribute to cardiovascular disease

c is because people with mental disorders do not understand healthy eating and the benefit of exercise

$d$ is due to a combination of factors, including lifestyle, economic disadvantage, unhelpful health behaviours and psychotropic drugs

$\mathrm{e}$ is because people with mental disorders do not want to stop smoking.

\section{Parity of esteem is:}

a a pledge that recognises and aims to address inequalities between physical and mental healthcare

b a guarantee that there will be more nurses and doctors to care for people with mental disorders

c a focus on training mental health professionals to recognise medical disorders

$\mathrm{d}$ an exercise to reduce waiting times for treatment of mental disorders e a law in the UK to ensure that people with mental disorders get equal care to those with physical disorders.

3 NICE recommends monitoring of physical health for people with established psychosis. In the first year of a psychotic illness, monitoring should be by:

a the GP or practice nurse

b the mental healthcare professionals treating the psychotic illness

c unspecified professionals

d any healthcare professional in contact with the patient

e health trainers.

4 Regarding lifestyle and severe mental illness, research has shown that:

a people with SMI have broadly the same rate of smoking as aged-matched population controls

b the majority of people with SMI are not interested in lifestyle intervention programmes

c lifestyle programmes do not improve risk factors in people with SMI in the short or long term

d drug-naive people with SMI have normal rates of smoking and hypertension e successful programmes to assist people with SMI to improve lifestyle tend to have multiple strategies administered over a sustained period.

5 Training and workforce planning is one of the recommendations of the Mental Health Taskforce to the NHS in England. For new and existing NHS-funded staff, this should include:

a cognitive-behavioural therapy in combination with improving access to psychological therapies (IAPT)

b basic mental health awareness, compassion and communication skills, mental illness prevention, and empowering people to understand their own strengths and to use self-help

$c$ a recognised qualification and endorsement from the Royal College of Psychiatrists

$\mathrm{d}$ a certificate in communication skills training

e access to a newly developed online parity of esteem course endorsed by the Royal College of Psychiatrists and the Royal College of Physicians. 